\title{
Social competence: an evolutionary approach
}

\author{
Barbara Taborsky ${ }^{\mathbf{1}}$ and Rui F. Oliveira ${ }^{2,3}$ \\ ${ }^{1}$ Behavioural Ecology, Institute of Ecology and Evolution, University of Bern, Wohlenstrasse 50A, \\ $\mathrm{CH}-3032$ Hinterkappelen, Switzerland \\ ${ }^{2}$ Instituto Superior de Psicologia Aplicada, Unidade de Investigação em Eco-Etologia, Rua Jardim do Tabaco 34 , \\ 1149-041 Lisboa, Portugal \\ ${ }^{3}$ Champalimaud Neuroscience Programme, Instituto Gulbenkian de Ciência, Rua da Quinta Grande 6, 2780-156 Oeiras, Portugal
}

\begin{abstract}
'Social competence' refers to the ability of an individual to optimise its social behaviour depending on available social information. Although such ability will enhance social interactions and thus raise Darwinian fitness, its evolutionary and ecological significance has been largely ignored. Social competence is based on behavioural flexibility. We propose that the study of social competence requires an integrative approach that aims to understand how the brain translates social information into flexible behavioural responses, how flexibility might be constrained by the developmental history of an individual or by trade-offs with other (ecological) competences, and how social plasticity feeds back on fitness. Finally we propose a hypothesis of how social competence can become a driver of social evolution.
\end{abstract}

\section{Behavioural flexibility as phenotypic plasticity}

Adaptation to the environment is a universal characteristic of living systems. According to classic evolutionary theory, adaptation by natural selection relies on heritable phenotypic variation produced by genetic variation. However, when the rate of genetic evolutionary change is outpaced by changes in the environment the need for adaptive change without genetic mutation emerges [1]. In this scenario, the evolution of phenotypic plasticity is favoured, that is, a certain genotype produces different phenotypes depending on environmental conditions [2]. Among animals, behavioural traits exhibit a greater plasticity than morphological and physiological traits and plastic changes are reversible within an individual's lifetime ('behavioural flexibility'). This makes behavioural flexibility a powerful, immediate mechanism allowing organisms to adapt to changing environmental conditions, which may or may not be followed by other flexible adjustments of physiology or morphology. Many of these responses are simple reflexes and fixed action patterns elicited by a stimulus in the environment, when it deterministically predicts an appropriate response. However, when environmental complexity and variability increase, the capacity to adaptively modify behaviour as a function of experience and context is needed. Although some degree of context-dependent behavioural flexibility may be achieved with genetically determined rules, behavioural flexibility will often depend on cognitive abilities (understood as the acquisition, retention, and use of information; [3]) that allow individuals to adapt behavioural output to specific situations in a complex and variable world (e.g., see [4]).

Interestingly, the evolutionary study of behavioural flexibility has rarely been framed within the scope of phenotypic plasticity, but rather in terms of cognitive evolution and ecology [3,5]. This is most probably due to the fact that in contrast to morphological and life history traits (which have been the main focus of phenotypic plasticity studies, and whose plasticity results from processes during development and is usually non-reversible) behavioural flexibility involves rapid changes, is labile, and is present during the whole life of the animal $[6,7]$.

\section{Glossary}

Behavioural reaction norm (BRN): the set of behavioural phenotypes that a single individual produces in a given set of environments [8]. This is in contrast to 'reaction norms' describing typically irreversible, developmental plasticity of a single genotype. Thus BRNs describe fast responses (within a life time) by an individual to variation in the environment.

Developmental plasticity: variation in the traits of individuals that results from processes during development as a consequence of environmental variation and that is typically irreversible [6].

Epigenetic modifications: changes in gene function that do not involve a change in the coding-sequence. Examples of such changes are DNA methylation and histone acetylation, both of which modify genome-regional gene expression without changing the sequence of the affected genes. Immediate early genes (IEGs): genes that show rapid and transient expression in response to a wide variety of extra-cellular stimuli and in the absence of $d e$ novo protein synthesis. Their products act as transcription factors on late response genes or as effectors that change the functioning of the cell, and thus they represent the first genomic response to stimuli [60].

Neural plasticity: structural and functional changes of the nervous system as a result of input from the environment. Two major mechanisms of neural plasticity operate at different time scales: structural rewiring of neural circuits is slow and long-lasting and induces dramatic behavioural changes, whereas biochemical modulation of existing neural networks is postulated to mediate fast and transient changes between motivational states that promote gradual changes in behavioural expression.

Neurogenomic state: transcriptome profile of the brain areas of interest for a given behavioural state (e.g., expression of a given social phenotype).

Phenotypic (behavioural) flexibility: changes of the (behavioural) phenotype that can be reversed within an individual's life time [6].

Phenotypic (behavioural) plasticity: an umbrella term subsuming different classes of plasticity, including developmental plasticity, phenotypic flexibility, and life-cycle staging [6].

Social competence: the ability of an animal to optimise the expression of its social behaviour as a function of the available social information.

Social context: any social stimulus that can vary across a gradient (e.g., group composition, offspring begging, or competitor density) [8].

Social information: any information that is generated by the behaviour of another organism. 
However, a phenotypic plasticity approach to behavioural flexibility would provide a new and powerful framework to understand the adaptive nature and evolution of animal behaviour; namely by introducing the concept of 'behavioural reaction norms' (BRN; see Glossary) as a tool to visualise and quantitatively analyse individual plasticity, and to unravel individual by environment $(\mathrm{I} \times \mathrm{E})$ interactions underlying it [8]. For example, recently this approach facilitated the establishment of a single framework to integrate the study of animal personality and behavioural plasticity, two phenomena usually studied separately, thereby enhancing the understanding of their adaptive significance [8]. This approach will also help to unravel the proximate mechanisms of behavioural flexibility, by extending the framework used to study the causes of phenotypic plasticity to behaviour (e.g., $[2,9]$ ).

\section{Adaptive behavioural flexibility in the social domain}

The social domain is arguably the most complex and fluctuating component of an animal's environment as it involves interaction with other behavioural agents with inherently associated higher levels of unpredictability. An animal interacting with its non-social abiotic or biotic environment will often modify this environment, thereby creating ecological feedback on the individual itself forcing it to flexibly adjust its behaviour (e.g., a foraging individual changes the local resource density enforcing an adjustment of subsequent foraging decisions). Such ecological feedback is ubiquitous and can even span generations (e.g., [10]), but nevertheless the degrees of freedom of this feedback will be finite. In contrast, feedback in the social domain can have nearly infinite degrees of freedom, as feedback will depend not only on social context but also on the number, identity, and the external (e.g., rank and size) and internal states of the agents that social behaviour is directed towards. Consequently, dealing with social complexity requires the evolution of cognitive mechanisms that allow the individual to assess the internal ('emotional') state of other organisms and the social context, and to integrate and process these stimuli not just as a result of direct effects of perceptual information, but rather as a function of what that perceptual information means to the individual at that moment in time [11]. Therefore, social decision-making depends on some kind of social experiential knowledge that allows organisms to evaluate stimuli and to determine the appropriate behaviour. Thus, more than in any other behavioural domain it is expected that social behaviour should exhibit high levels of plasticity.

The ability of individuals to regulate the expression of their social behaviour in order to optimise their social relationships is referred to as 'social competence'. It should be stressed here that flexibility in the expression of social behaviour is necessary but not sufficient for social competence, since the latter implies not only variation in the response to the same social stimuli depending on additional social information, but also the demonstration that this variation is adaptive (i.e., optimises the response). Social competence allows organisms to express appropriate responses to demands and to generate and capitalise on opportunities in the social environment [12] thereby ultimately enhancing their fitness. For example,

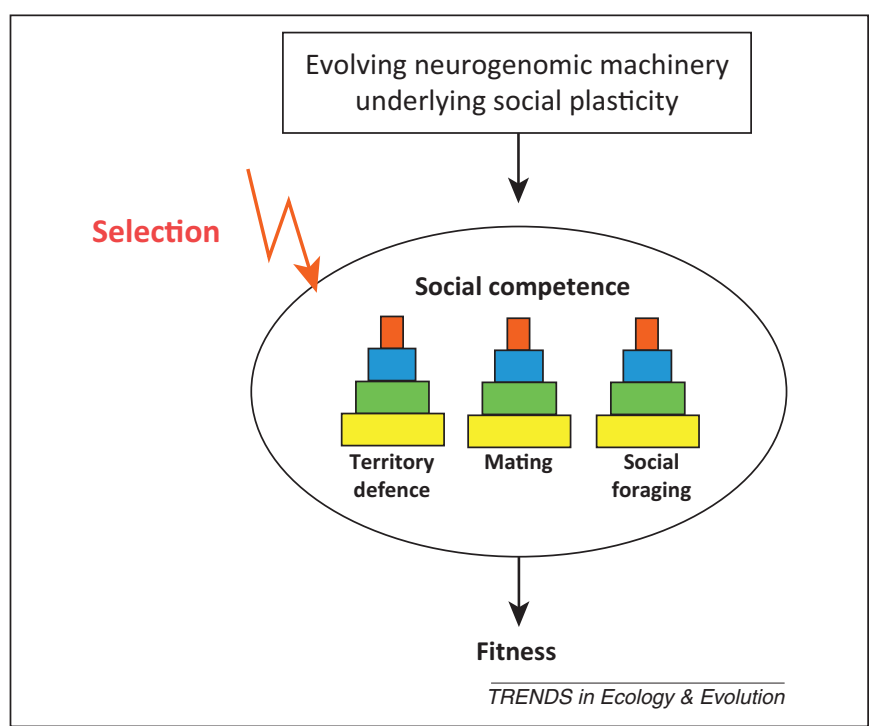

Figure 1. Social competence is hypothesised to be an ability underlying all social behaviour that is contingent on social information and thus to affect behaviour in many or all social contexts. This should give rise to positive within-individual correlations of social performance across different social contexts; social performance is under positive selection and affects fitness. Differently coloured rectangles represent different individuals; the width of these rectangles represents an individual's social performance in the different social contexts.

social competence will enable individuals to avoid engaging in costly social interactions or being ejected from their social groups.

Social competence has been shown to influence the performance of different social behaviours across different social contexts (Box 1). There are two possible scenarios that can explain this joint effect. Either social competence is composed of a collection of social skills, each of which evolves rather independently, or, alternatively, social competence may be an ability underlying all social subdomains (e.g., interactions with competitors, mating, and social foraging) resulting in positive within-individual correlations of performance across different social contexts (Figure 1). Although some evidence supports the former hypothesis (e.g., independence of social skills that contribute to mating success in cowbirds, such as song quality, courtship persistence, and competitive ability [13]) most of the available evidence supports the second alternative, namely the fact that the social environment experienced during ontogeny can affect the appropriateness of suites of social behaviours belonging to various different social contexts [14-17].

\section{Animal social competence}

To date the study of social competence has been mainly a domain of the social sciences, with a strong focus on causal relationships between social factors and the development and expression of social competence in humans [12,18,19]. However, indications of social competence are also well known from non-human animals. Interacting animals respond to the presence of bystanders ('audience effect') by changing their signalling behaviour according to the type of audience and social context (e.g., [20,21]). By contrast, bystanders extract information from observed interactions ('eavesdropping') that they use in subsequent interactions 


\section{Box 1. How to measure social competence}

If we want to study individual variation in social competence, we need an approach that captures the universal nature of this trait. We propose to decide upon a representative, ecologically relevant set of social situations, of which we understand the adequacy of the possible involved behaviours. We should expose individuals to these situations in standardised trials testing them: (i) in different social contexts (e.g., dominance relationships, mating, and brood care) to test for the presence of a general social ability; (ii) in different social roles (e.g., dominant and subordinate) within the same context to test if this ability is based on plasticity; (iii) in an unknown social situation (e.g., an ecologically relevant situation that had not yet been encountered during ontogeny) to check for the ability to generalise across social situations; (iv) at different life stages to test if individual differences in social competence persist over time. It is important to have a priori predictions about which social behaviours will be optimal in a given test. Classifying those behaviours as socially competent that are expressed by individuals with the highest Darwinian fitness may be misleading, because a high fitness may result from competences outside the social domain (e.g., solitary foraging ability).

We illustrate this research agenda by an example from the cooperatively breeding cichlid Neolamprologus pulcher (Figure I).
These fish had been reared either with or without the presence of older conspecifics to investigate whether experiencing a more complex social environment results in better social competence $[16,17]$. Three challenge tests (T1-T3) were conducted to assess social competence along the four above mentioned axes (Table I). In T1 juveniles were assigned either the ownership of a critical resource (a shelter) or of an intruder (asymmetric competition). In nature, subordinate group members defend private shelters within the breeders' territory against other group members. Shelter access can decide over survival as predation pressure is intense. The intruder role reflects the situation of a juvenile subordinate in search of an own private shelter after it is no longer allowed to access the natal breeding cavity. T2 simulated a symmetric contest over a shelter, in which both opponents had been assigned the role of the shelter owner prior to the test. This mimics a situation in which a shelter has to be defended against an at least equally motivated opponent (symmetric competition). In T3 larger, adult subordinates were forced to achieve acceptance at the territory of an unfamiliar breeder pair, a situation fish would encounter after dispersal from the natal territory [74]. In this situation subordinates should strive for acceptance as brood care helpers.
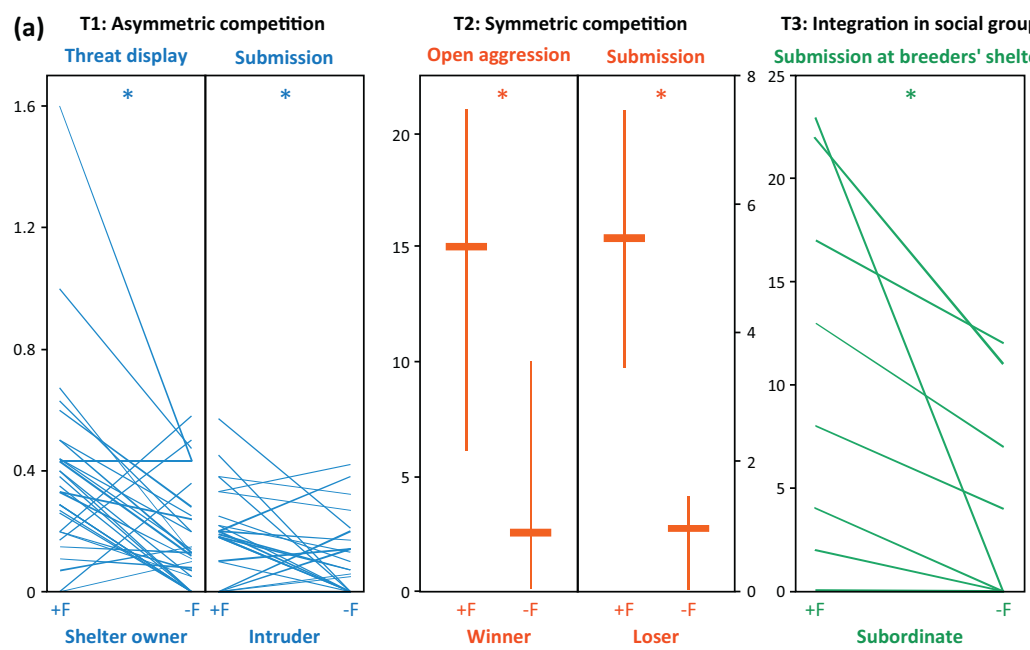

(b)

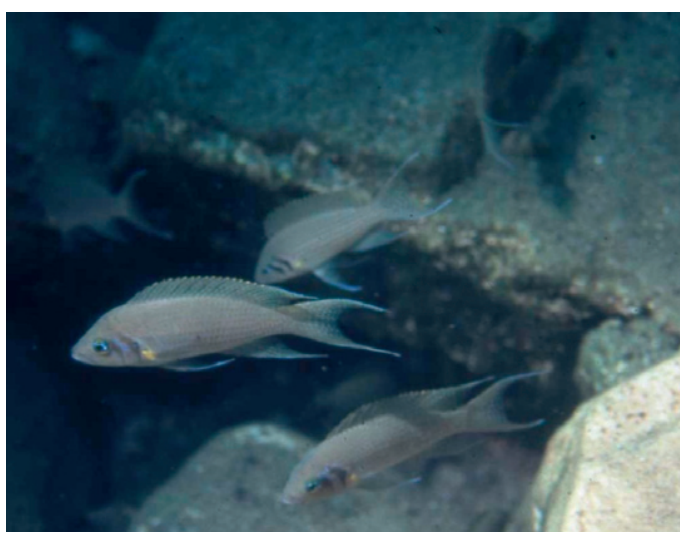

$\overline{T R E N D S}$ in Ecology \& Evolution

Figure I. (a) Neolamprologus pulcher that had been reared together with older conspecifics showed more of the expected appropriate behaviours in an asymmetric competition test (blue), a symmetric competition test (red), and a previously unknown social challenge, namely being exposed to an unfamiliar breeder pair (green). (b) Neolamprologus pulcher lives and breeds in social groups of 3-38 individuals that are structured by a size-dependent dominance hierarchy. Related and unrelated smaller individuals help the dominant breeder pair rearing their offspring.

Table I. Three behavioural tests to assess the differences in social competence between $N$. pulcher reared with or without older conspecifics (parents and/or helpers). In all tests, fish reared with older conspecifics showed significantly more of the behaviours predicted to be appropriate in a given situation (Figure I), whereas no significant differences were found in behaviours predicted to be inappropriate $[16,17]$

\begin{tabular}{|c|c|c|c|c|c|c|}
\hline Challenge test & (i) Social context & $\begin{array}{l}\text { (ii) Different roles } \\
\text { within a context }\end{array}$ & $\begin{array}{l}\text { (iii) Is the } \\
\text { situation } \\
\text { known? }\end{array}$ & (iv) Life stage & $\begin{array}{l}\text { Predicted } \\
\text { appropriate } \\
\text { behaviour }\end{array}$ & $\begin{array}{l}\text { Predicted } \\
\text { inappropriate } \\
\text { behaviour }\end{array}$ \\
\hline \multirow[t]{2}{*}{$\mathrm{T} 1$} & Competition & Owner & Yes & Early juvenile & Threat display & $\begin{array}{l}\text { Open aggression, } \\
\text { submission }\end{array}$ \\
\hline & & Intruder & & & Submission & Escape \\
\hline \multirow[t]{2}{*}{$\mathrm{T} 2$} & Competition & Winner & Yes & Late juvenile & Open aggression & $\begin{array}{l}\text { Threat display, } \\
\text { submission }\end{array}$ \\
\hline & & Loser & & & Submission & Escape \\
\hline T3 & $\begin{array}{l}\text { Integration by } \\
\text { subordinate } \\
\text { in a new group }\end{array}$ & - & No & Adult & $\begin{array}{l}\text { Submission near } \\
\text { prospective breeding } \\
\text { cavity }\end{array}$ & Aggression, escape \\
\hline
\end{tabular}


with the observed individuals [22,23]. Familiarity with an opponent is also known to reduce ('dear enemy effect' [24]) or increase ('nasty neighbour effect' [25]) fighting behaviour depending on the relative threat imposed to territory holders by neighbours versus strangers. Previous social experience can affect subsequent behaviour as in the case of 'winner-loser effects' demonstrated across different animal taxa [26,27], where previous winners are more likely to win successive contests, and losers will more likely lose again even against different opponents. A similar effect is known from a cooperative context where individuals give more help to social partners if they had received help by another conspecific before ('generalised reciprocity' [28]). Experiments have shown that Norway rats optimise their social behaviour as a function of social information obtained in previous fights, as acting contingently on this information increased an actor's fitness. Previous winners won successive fights after a shorter time despite a reduced amount of aggressive behaviour, and losers faced a decreased risk of injury despite a reduced amount of submissive behaviour shown [29]. Most of the abovementioned effects of social information on behaviour have been observed to be widespread in natural populations $[24,25,27,30]$ and their existence was confirmed by targeted field experiments [21,24-27]. Thus we propose that social competence allows animals to efficiently navigate the complexities of their social environment in order to survive, reproduce, and raise their offspring, and therefore it should be seen as a key determinant of the Darwinian fitness of individuals.

\section{Social competence versus general cognition}

Since social competence can give rise to consistent expression of appropriate flexible behavioural responses across different social contexts, it appears similar to the concept of general intelligence. In humans, performance on diverse cognitive tasks shows robust positive correlations, so that individuals scoring high on one cognitive ability are also likely to score well in others. This positive manifold of correlations has been interpreted as being caused by a latent single factor named general intelligence, or simply 'g' [31]. In comparative cognition it has been debated whether cognition is organised into separate modules or whether there is a general problem-solving ability that pertains all behavioural domains and ecological demands. Although comparative analyses, mainly focused on primates and birds, suggest the lack of cognitive modules and support a general intelligence scenario [32-35], experimental approaches in single species produce conflicting results. Different developmental studies show that early social experience can promote social competence and social learning, but leaves the performance in non-social cognitive tasks unaffected. For example, after maternal deprivation, laboratory rats performed more poorly than normally-reared rats in three social learning tasks, whereas in two spatial learning tasks the treatment groups performed equally well [36]. Similarly, communallyreared mice had a better social competence during their adult life than single-mother reared mice, but they did not differ in spatial learning and memory capacity tests $[37,38]$. Zebrafish reared among more diverse social partners (mixed strains versus single strain) were more aggressive later in life, but non-social behaviours such as general activity, predator evasion behaviour and stress recovery were unaffected [39]. Finally, in humans intelligence quotients (IQ) and social intelligence tests for assessment of the theory of mind were not correlated [40]. Part of these contradictory results may be explained by the interpretation of comparative studies that have identified a species-level composite general intelligence factor. As for human intelligence, it is important to distinguish between 'g' as a general first-order factor or dominant eigenvalue in a factor analysis, from 'g' as a psychological construct that reduces cognitive abilities to a single quantitative cognitive or biological process [41]. Alternative models have been proposed that illustrate how a psychometric ' $g$ ' can be explained by dynamic interactions between cognitive processes during development, rather than by a single underlying process [41]. Together, these results suggest that it is unlikely that social competence is a subdomain of general intelligence, but further studies are needed to clarify this issue.

\section{An evolutionary framework for the study of social competence}

If we understand social competence as a general ability affecting individual performance in a social environment, it should have the key properties of an ecological performance trait [42], namely (i) the existence of inter-individual variation in performance ranging from low to high, and (ii) this variation should be positively correlated with fitness [43]. Furthermore, for it to be an evolvable trait, potential evolutionary costs, benefits, and trade-offs of social competence should also be identified.

(i) Variation in performance

Persistent individual variation in social performance can be due to genetic disposition or it can be induced during ontogeny. Genetic disposition has so far been addressed only in humans: twin studies have detected significant heritability of social competence as assessed by questionnaires ([44,45] and references therein). A much larger body of literature has shown that variation in social competence can be environmentally induced and originates from developmental plasticity (Table 1). However, only a few of these studies tested performance across different contexts. For example, communally-reared mice exposed to more social contacts showed more appropriate social behaviours in the contexts of dominance interactions and brood care, compared to single-mother reared mice [15]. Also, cichlid fish (Neolamprologus pulcher) reared by alloparents behaved more appropriately in a competitive context or when striving for the acceptance as helper by a breeder pair, than individuals reared among siblings only (Box 1, $[16,17])$.

(ii) Correlation with fitness

Several studies, which had induced differences in social performance by manipulations of social cues during development, found associated differences in fitness correlates (Table 1). For example, in some rodents, being exposed to a more complex social 


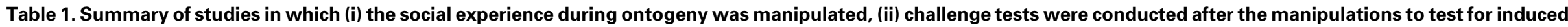
differences in social performance, and (iii) effects of differential social behaviour on fitness correlates were reported

\begin{tabular}{|c|c|c|c|c|c|c|}
\hline Species & Rearing environment & $\begin{array}{l}\text { Life stage of } \\
\text { experience }\end{array}$ & $\begin{array}{l}\text { Social context of } \\
\text { challenge test }\end{array}$ & Phenotypic effect ${ }^{a}$ & Effects on fitness ${ }^{a}$ & Refs \\
\hline \multirow[t]{3}{*}{ Neolamprologus pulcher } & $\begin{array}{l}\text { Parents and siblings vs } \\
\text { siblings-only }\end{array}$ & Dependent young & Competition & $\begin{array}{l}\text { More appropriate use of aggression } \\
\text { and submission }\end{array}$ & Shorter fights & [16] \\
\hline & & & Competition & $\begin{array}{l}\text { More appropriate use of aggression } \\
\text { and submission }\end{array}$ & Losers less often evicted & [17] \\
\hline & & & $\begin{array}{l}\text { Integration in } \\
\text { social group }\end{array}$ & $\begin{array}{l}\text { More submissive behaviour at } \\
\text { breeding cavities }\end{array}$ & Higher tolerance by breeders & [17] \\
\hline $\begin{array}{l}\text { Zebra finch, Taeniopygia } \\
\text { guttata }\end{array}$ & $\begin{array}{l}\text { With vs without males } \\
\text { present in breeding colony }\end{array}$ & Dependent young & Mate choice, mating & Prefer opposite sex over same sex & Higher mating success & [79] \\
\hline Cowbirds, Molothrus ater & Dynamic vs static flock & Adult males & Mate choice, mating & Larger and more variable 'singing network' & Higher mating success & [13] \\
\hline Lab mouse, CD1(ICR) & $\begin{array}{l}\text { Communal vs single-mother } \\
\text { rearing }\end{array}$ & Dependent young & $\begin{array}{l}\text { Dominance } \\
\text { interactions }\end{array}$ & Higher propensity to interact socially & $\begin{array}{l}\text { Shorter time to find hierarchy } \\
\text { position }\end{array}$ & [14] \\
\hline Lab mouse, CD1(ICR) & $\begin{array}{l}\text { Synchronous vs asynchronous } \\
\text { birth-spacing in communal nest }\end{array}$ & Dependent young & $\begin{array}{l}\text { Dominance } \\
\text { interactions }\end{array}$ & Higher aggression/lower affiliative behaviour & $\begin{array}{l}\text { Shorter time to find hierarchy } \\
\text { position }\end{array}$ & [80] \\
\hline Lab mouse, CD1(ICR) & Mixed sex vs female only litter & Dependent young & Maternal care & $\begin{array}{l}\text { Shorter pup retrieval latency, } \\
\text { higher aggression towards intruder males }\end{array}$ & Better maternal care & [81] \\
\hline \multirow[t]{2}{*}{ Lab mouse, Balb/c } & $\begin{array}{l}\text { Communal vs single-mother } \\
\text { rearing }\end{array}$ & Dependent young & Maternal care & $\begin{array}{l}\text { Shorter pup retrieval latency, more care, } \\
\text { better nest quality }\end{array}$ & $\begin{array}{l}\text { Better maternal care, F2 had } \\
\text { larger litters }\end{array}$ & [15] \\
\hline & & & $\begin{array}{l}\text { Dominance } \\
\text { interactions }\end{array}$ & Lower aggression towards intruder males & & \\
\hline Lab rat, Porton & Mixed sex vs female only litter & Dependent young & Maternal care & $\begin{array}{l}\text { More elaborate nests and more building } \\
\text { behaviour }\end{array}$ & Fewer and smaller litters produced & [82] \\
\hline \multirow[t]{2}{*}{ Lab rat, Sprague-Dawley } & With vs without mother present & Dependent young & 3 social learning tests & Enhanced social learning ability & & [36] \\
\hline & & & $\begin{array}{l}\text { Short separation } \\
\text { from pups }\end{array}$ & Shorter latency to show maternal care & Better maternal care & \\
\hline $\begin{array}{l}\text { Oldfield mouse, } \\
\text { Peromyscus polionotus }\end{array}$ & $\begin{array}{l}\text { With vs without } \\
\text { younger-sibling pups }\end{array}$ & Subadult daughters & Mating, reproduction & Better nest quality & Higher reproductive success & [46] \\
\hline $\begin{array}{l}\text { Rhesus macaque, } \\
\text { Macaca mulatta }\end{array}$ & With vs without mother present & Dependent young & $\begin{array}{l}\text { Staging intra-group } \\
\text { conflict }\end{array}$ & Better in acquisition of dominance rank & Higher rank achieved & [83] \\
\hline
\end{tabular}

'Directions of the reported results refer to the respective treatment group that was exposed to the more complex social condition, and that is the first condition mentioned in the column 'Rearing environment'. 
environment during ontogeny resulted in better maternal care behaviour and higher reproductive success for females or their daughters $[15,46]$.

(iii) Benefits of social competence

Expressing a better social competence should generally be beneficial in all kinds of social encounters, and the majority of animal species will be involved in at least some social encounters. The expected benefits of social competence will be particularly strong in socially living species, where behaviours involved in vital functions such as acquiring and defending resources, predator evasion, and rearing offspring will usually involve social interactions and/or occur in socially heterogeneous environments (e.g., in environments where individuals face quickly changing social contexts and a diverse array of social interactions and relationships).

(iv) Costs of social competence

There will be costs associated with developing and maintaining the sensory and neural machinery necessary for processing social information that influences social behaviour [47]. Arguably, the cognitive demands of a social life selected for larger brains that are metabolically expensive (i.e., social brain hypothesis [48,49]), although brain size appears to be associated with quantitative rather than qualitative improvements in cognitive abilities [50]. Learning and memory capacity involved in the correct interpretation of social information will often involve both time and energy costs [51,52]. Additionally, production costs may arise when costly behaviours are involved in a socially competent response [47]. For example, the appropriate response towards agonistic displays by dominants of the cichlid fish $N$. pulcher is a submissive display by subordinates, which raises the energy expenditure by roughly five times the standard metabolic rate [53]. (v) Possible trade-offs

Given the set of possible costs associated with acquiring and maintaining social competence, trade-offs with other body functions are expected on various levels. Obtaining social information needed for an accurate social response increases sampling time and thus compromises the speed of social decision-making, especially under noisy conditions (i.e., speed-accuracy trade-off in decision making [54]). Time needed during early ontogeny to learn interpreting social stimuli and expressing social behaviour correctly [16] might conflict with the time required to learn about other important ecological stimuli (shelters, predator evasion skills, or foraging techniques). Finally, genetic trade-offs may arise from an involvement of learning in the acquisition of social competence, as suggested by the existence of a genetic trade-off between learning ability and competitive ability in fruit fly larvae [55,56].

\section{Integrating proximate mechanisms in the evolutionary study of social competence}

The idea that social competence - like any other adaptive behaviour - relies on optimal behavioural rules is unrealistic, since its proximate mechanisms will impose constraints and limits to flexible behavioural responses $[57,58]$. Being a plastic trait, social competence relies on the generation of multiple social phenotypes from the same genotype, a process that is expected to result from interactions between genetic, environmental, and epigenetic processes that lead to neural and behavioural plasticity [2]. This involves the regulation of genome-wide gene expression in the nervous system (i.e., brain transcriptome) by social information, which can be achieved by two different mechanisms at different time scales. Epigenetic modifications (e.g., DNA methylation and histone

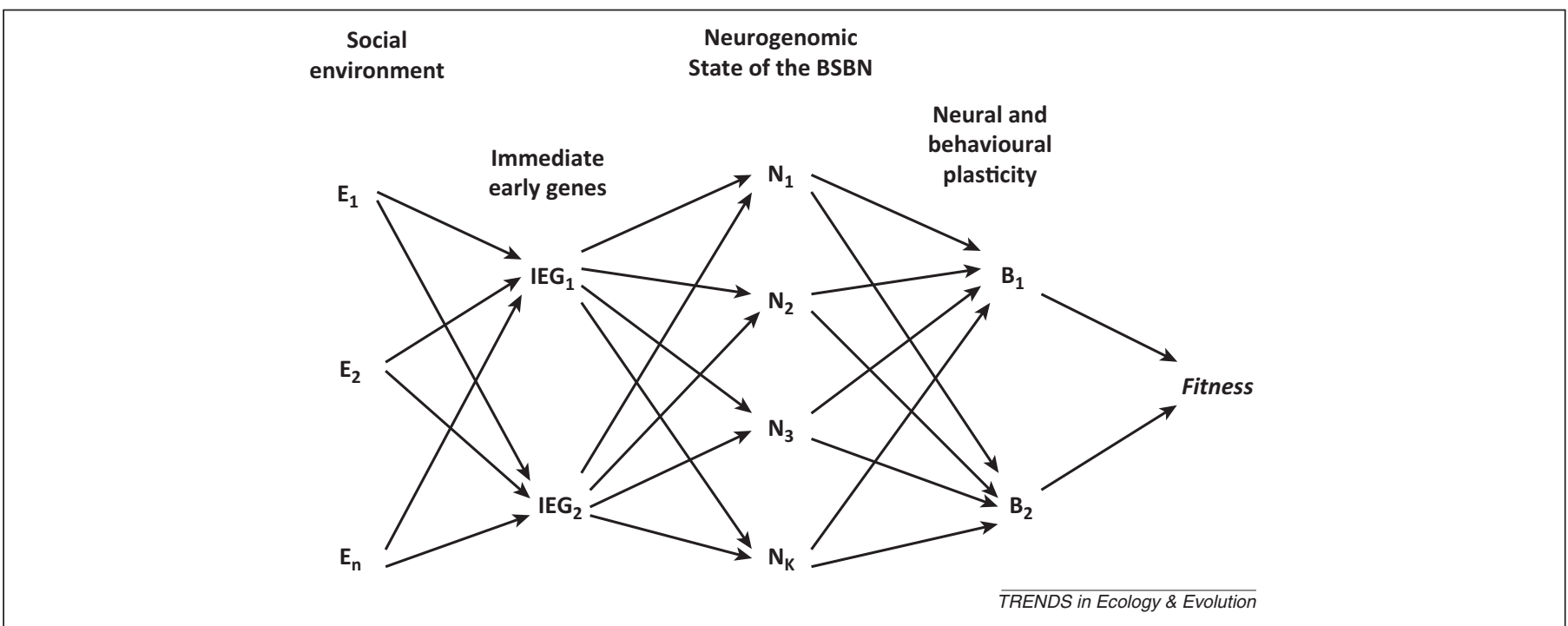

Figure 2. Path diagram showing the proposed relationship between the social environment (E1-En), immediate early genes (IEG1 and IEG2), neurogenomic states of relevant neural circuits (N1-Nk), that will determine the neural and behavioural plasticity that in turn translates in the expression of a specific behavioural profile (B1 and B2), with implications for the Darwinian fitness of the animal. Variations in the social environment induce changes in IEG expression that will orchestrate the plasticity of the neural circuits underlying social behaviour that have fitness consequences. 


\section{Box 2. Molecular mechanisms of adaptive behavioural flexibility}

At the proximate level behavioural flexibility is achieved by rewiring or by biochemically switching nodes of the neural network underlying social behaviour (i.e., 'Brain Social Behaviour Network' or BSBN [75]) in response to perceived social information (Figure I). Therefore, at the molecular level, it depends on the social regulation of gene expression so that different neurogenomic states correspond to different behavioural responses and the switches between states are orchestrated by signalling pathways that interface the social environment and the genotype. Transient socially-driven neuroplasticity can be achieved by three different neuronal-activity dependent mechanisms [2,76]: (a) activation (e.g., phosphorylation) of proteins that then act as transcription factors for IEGs (e.g., CREB) or for delayed response genes (DRGs) or regulate intracellular signalling pathways (e.g., MAPKs); (b) neuronal activity-dependent transcription factors (e.g., pCREB) activate immediate early genes (IEG) that can encode other transcription factors (e.g., c-Fos and Egr-1) or synaptic proteins (Arc and Homer1a), hence acting as neuromolecular switches that change the expression of co-regulated gene sets in the brain; (c) transcription of microRNAs that regulate translation of synaptic proteins (e.g., miR-134). By contrast, socially-driven long-lasting changes in social behaviour rely on epigenetic modifications (e.g., DNA methylation and histone modifications) of genes involved in social behaviour (e.g., oxytocin and vasopressin) or neural plasticity (e.g., bdnf and npas4) [77,78]). Together, these neuronal-activity dependent mechanisms change the neurogenomic state of the brain in response to perceived social stimuli. These changes in gene expression may occur differentially across the different nodes of the neural network underlying the expression of social behaviour. Neural circuits underlying behaviour are composed by a network of brain nuclei with reciprocal connections between each pair that encodes information in a distributed and dynamic fashion, such that the expression of a given behaviour is better reflected by the overall profile of activation across the different loci in the network than by the activity of a single node (e.g., BSBN). Different combinations of activation across nodes, and variation in the strength of the connections among them, will generate an almost infinite variation in social behaviour. Therefore, the changes in neurogenomic states mentioned in the previous point can occur differentially at each of the nodes of the BSBN, and social plasticity relies both on temporal and spatial changes in gene regulation in the neural networks.

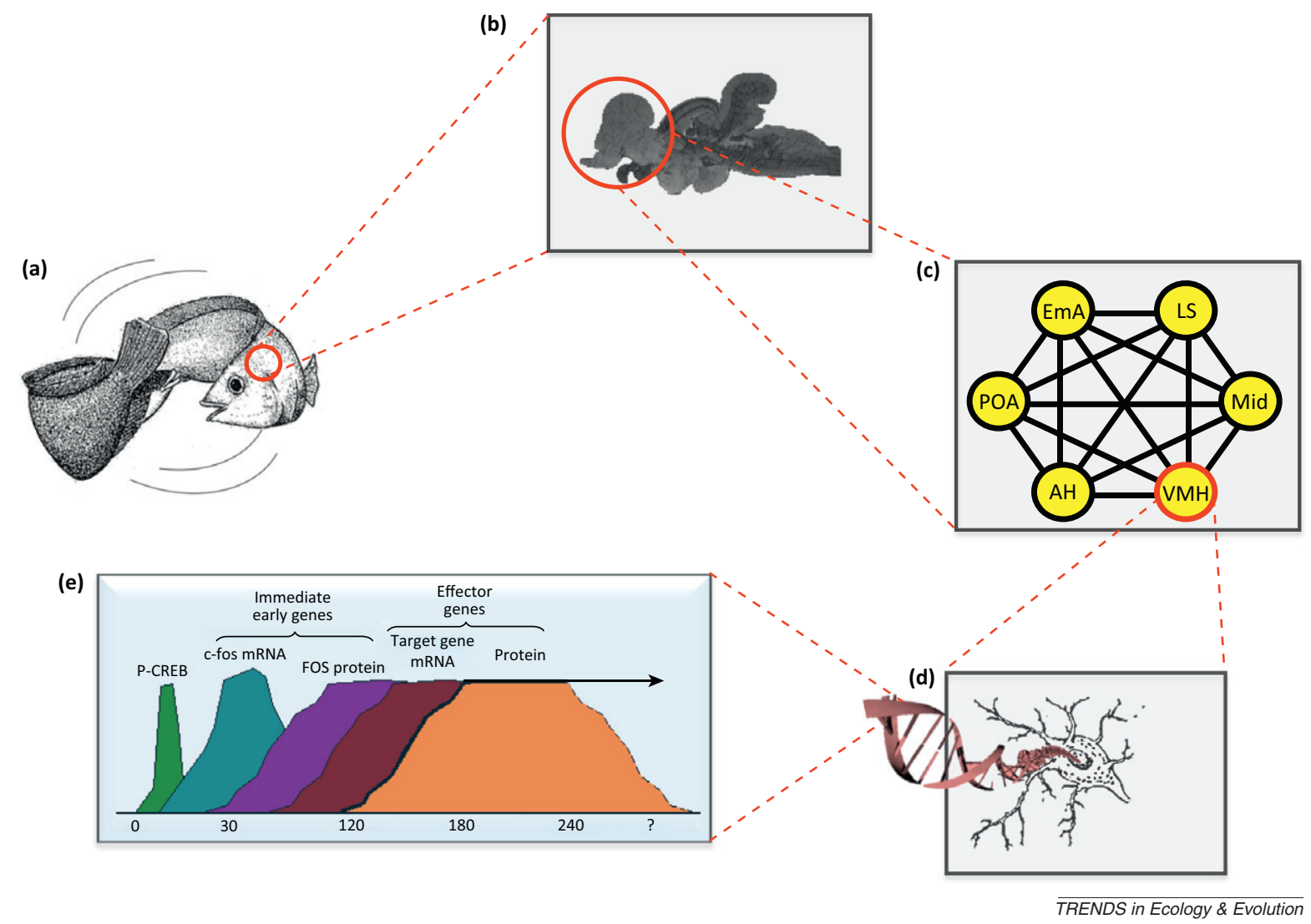

Figure I. Neural and molecular mechanisms of social competence: animals adjust the expression of their social behaviour according to previous social interactions and social context (a); social information will be encoded in a distributed brain social behaviour network (BSBN) (b); at each node of this network (c) neurons will change their neurogenomic state (d), that is, their gene expression profile in response to the perceived social information; changes of gene expression are triggered by the activation of neuronal activity-regulated transcription factors (e.g., pCREB) that regulate immediate early genes (e.g., $c$-fos) that can regulate synaptic proteins (e), therefore modulating neural plasticity that underlies behavioural flexibility. In this figure the nodes of the BSBN are named after the mammalian homologues of teleost brain areas (abbreviations: EmA = extended medial amygdala, $\mathrm{LS}=$ lateral septum, Mid = central gray in the midbrain, $\mathrm{VMH}=\mathrm{ventromedial}$ hypothalamus, $\mathrm{AH}=$ anterior hypothalamus, $\mathrm{POA}=$ pre-optic area).

modifications) provide a way for environmental effects to have a sustained effect on gene expression profile, and therefore they can explain life stage changes in social plasticity as well as inter-individual differences (e.g., 'behavioural profiles') resulting from early life conditions, whereas immediate-early gene (IEG) response accounts for short-term changes in neurogenomic states underlying behavioural flexibility [9,59]. IEG (e.g., c-fos, egr-1, and arc) expression does not require the activation of any preceding gene, and since IEGs modify synaptic structure and function, they represent the earliest genomic response to an inducing stimulus that orchestrates integrated 
genomic responses to social information. The wave of IEG activation following a stimulus is recruiting temporally correlated associations in neural activity in behaviourally significant contexts and promotes the slower alteration of synaptic networks, thereby adjusting the selectivity of longterm information storage and retrieval in neuronal networks [60]. Thus, temporal and spatial variation in gene expression in the brain regulates the remodelling of the neural networks that underlie behavioural flexibility. Some IEGs have been shown to be activated within minutes after exposure to specific social cues and to vary their activation with the valence (appetitive vs aversive) and salience (e.g., familiarity and complexity) of the social signal [61-63]. IEG activation has been documented in response to a wide range of social stimuli in different species and sensory modalities (e.g., songbirds [64], African cichlid fish [65], and Tungara frogs [66]), confirming the role of IEGs as neuromolecular switches for the transduction of social information into changes in brain function and behaviour. In response to IEG expression, co-regulated gene sets ('neurogenomic states') are then co-expressed leading to an association between behaviourally driven gene expression and the expression of social phenotypes (e.g., differences in transcriptome profiles between social phenotypes and contexts: hiveworkers vs foragers in honey bees [67], workers vs queens in ants [68], dominant vs subordinate fish and mammals [69,70], monogamous vs polygynous African cichlids [71], response to social vs sexual stimuli [72], and territoriality at different life-history stages [73]).

\section{The role of the Brain Social Behaviour Network (BSBN) in generating behavioural flexibility}

Knowledge of the proximate mechanisms underlying social competence is crucial to understanding the costs, limits, and evolutionary consequences of social plasticity, therefore enabling a better understanding of the dynamics of selection. We propose an integrative framework for future research in social competence that integrates proximate mechanisms into the study of evolutionary consequences (summarised in Figure 2). According to this approach, relevant social information induces changes in neuronal activity-dependent IEGs. Given their pivotal role in regulating gene networks, these IEGs will in turn activate massive temporal and spatial changes in gene expression across the different nodes of the neural network underlying social behaviour, the BSBN (Box 2), thereby orchestrating neural plasticity at the level of the network and generating behavioural flexibility. These flexible behavioural responses will enable individuals to navigate more efficiently their social environment at multiple domains (e.g., defending territories, finding mates, and establishing a position in a hierarchy) thereby impacting Darwinian fitness. Since spatio-temporal changes in the neurogenomic states of the BSBN will give raise to an almost unlimited number of behavioural plasticity states, this is a prime mechanism to generate diversity in social behaviour on which selection may act, selecting the combinations that produce adaptive behaviours. However, the number of combinations produced will be constrained by epigenetic as well as by pleiotropic and epistatic effects of the genes involved in neural plasticity at the BSBN level, imposing a limit to behavioural flexibility. Thus, the optimisation of behavioural flexibility (i.e., social competence) will be limited by behavioural consistency (i.e., personality, see [8]), and the two should be viewed as sharing common proximate mechanisms that should be taken into account when discussing their evolutionary implications.

\section{Concluding remarks}

Here we draw attention to the evolutionary importance of adaptive behavioural plasticity in the social domain and propose an integrated framework for its study that combines investigating proximate mechanisms and ultimate consequences. Placing social competence in an evolutionary framework will facilitate exploration of its evolvability and potential evolutionary consequences. For example, if variation between individuals exists in social competence, individuals with a slightly better social competence will tend to engage more often in social interactions, because they can effectively reduce costs of these interactions and therefore enjoy higher net benefits. Thus, these individuals would be relatively more social, which in turn will increase selection pressure on their social performance. By this positive feedback, selection on social competence may enhance the evolution of sociality.

The integrative approach proposed here to the study of social competence has the added value of using information on the molecular mechanisms of behavioural flexibility to get an insight into its evolutionary dynamics. Although we describe this approach for social plasticity, similar processes can be expected to be in place for other behavioural domains, since other neural networks underlying behaviour have been identified in vertebrates, and therefore the spatio-temporal regulation of gene expression in behavioural brain networks may be a general process to generate the neural plasticity needed to accommodate behavioural flexibility in general.

\section{Acknowledgements}

During the writing of this paper B.T. was funded by the Swiss National Science Foundation (SNF, Project 31003A_133066) and R.F.O. by the Portuguese Foundation for Science and Technology (FCT, Grants RGLVT-331-2352 and PTDC/PSI-PCO/118776/2010). We thank Ralph Bergmüller for productive conceptual discussions on this topic that prompted the writing of this paper, and Miguel Simões for helping with the preparation of Figure I in Box 2.

\section{References}

1 Frank, S.A. (2011) Natural selection. I. Variable environments and uncertain returns on investment. J. Evol. Biol. 24, 2299-2309

2 Aubin-Horth, N. and Renn, S.C. (2009) Genomic reaction norms: using integrative biology to understand molecular mechanisms of phenotypic plasticity. Mol. Ecol. 18, 3763-3780

3 Shettleworth, S.J. (1998) Cognition, Evolution and Behavior, Oxford University Press

4 Brosnan, S.F. et al. (2010) The interplay of cognition and cooperation. Philos. Trans. R. Soc. Lond. B: Biol. Sci. 365, 2699-2710

5 Reader, S.M. and Laland, K.N. (2002) Social intelligence, innovation, and enhanced brain size in primates. Proc. Natl. Acad. Sci. U.S.A. 99, 4436-4441

6 Piersma, T. and Drent, J. (2003) Phenotypic flexibility and the evolution of organismal design. Trends Ecol. Evol. 18, 228-233

7 Sih, A. et al. (2004) Behavioral syndromes: an integrative overview. Q. Rev. Biol. 79, 241-277

8 Dingemanse, N.J. et al. (2010) Behavioural reaction norms: animal personality meets individual plasticity. Trends Ecol. Evol. 25, 81-89 
9 Burmeister, S.S. (2007) Genomic responses to behavioral interactions in an African cichlid fish: mechanisms and evolutionary implications. Brain Behav. Evol. 70, 247-256

10 Laland, K.N. and Sterelny, K. (2006) Seven reasons (not) to neglect niche construction. Evol. Dev. 60, 1751-1762

11 Paul, E.S. et al. (2005) Measuring emotional processes in animals: the utility of a cognitive approach. Neurosci. Biobehav. Rev. 29, 469-491

12 Waters, E. and Sroufe, L.A. (1983) Social competence as a developmental construct. Dev. Rev. 3, 79-97

13 White, D.J. et al. (2010) The ontogeny of social skills: experimental increases in social complexity enhance reproductive success in adult cowbirds. Anim. Behav. 79, 385-390

14 Branchi, I. et al. (2006) Early social enrichment shapes social behavior and nerve growth factor and brain-derived neurotrophic factor levels in the adult mouse brain. Biol. Psychiatry 60, 690-696

15 Curley, J.P. et al. (2009) Social enrichment during postnatal development induces transgenerational effects on emotional and reproductive behavior in mice. Front. Behav. Neurosci. 3, 25

16 Arnold, C. and Taborsky, B. (2010) Social experience in early ontogeny has lasting effects on social skills in cooperatively breeding cichlids. Anim. Behav. 79, 621-630

17 Taborsky, B. et al. (2012) The early social environment affects social competence in a cooperative breeder. Anim. Behav. 83, 1067-1074

18 Dodge, K. (1985) Facets of social interaction and the assessment of social competence in children. In Children's Peer Relations: Issues in Assessment and Intervention (Schneider, B. et al., eds), pp. 3-22, Springer

19 Rose-Krasnor, L. (1997) The nature of social competence: A theoretical review. Soc. Dev. 6, 111-135

20 Doutrelant, C. et al. (2001) The effect of an audience on intrasexual communication in male Siamese fighting fish, Betta splendens. Behav. Ecol. 12, 283-286

21 Pinto, A. et al. (2011) Cleaner wrasses Labroides dimidiatus are more cooperative in the presence of an audience. Curr. Biol. 21, 1140-1144

22 Oliveira, R.F. et al. (1998) Know thine enemy: fighting fish gather information from observing conspecific interactions. Proc. R. Soc. Lond. B: Biol. Sci. 265, 1045-1049

23 Earley, R.L. (2010) Social eavesdropping and the evolution of conditional cooperation and cheating strategies. Philos. Trans. $R$. Soc. Lond. B: Biol. Sci. 365, 2675-2686

24 Temeles, E.J. (1994) The role of neighbours in territorial systems: when are they 'dear enemies'? Anim. Behav. 47, 339-350

25 Müller, C.A. and Manser, M.B. (2007) 'Nasty neighbours' rather than 'dear enemies' in a social carnivore. Proc. R. Soc. Lond. B: Biol. Sci. 274, 959-965

26 Rutte, C. et al. (2006) What sets the odds of winning and losing? Trends Ecol. Evol. 21, 16-21

27 Hsu, Y. et al. (2006) Modulation of aggressive behaviour by fighting experience: mechanisms and contest outcomes. Biol. Rev. 81, 33-74

28 Rutte, C. and Taborsky, M. (2007) Generalized reciprocity in rats. PLoS Biol. 5, e196

29 Lehner, S.R. et al. (2011) Rats benefit from winner and loser effects. Ethology 117, 949-960

30 Bshary, R. and Grutter, A.S. (2002) Asymmetric cheating opportunities and partner control in a cleaner fish mutualism. Anim. Behav. 63, 547555

31 Neisser, U. et al. (1996) Intelligence: knowns and unknowns. Am. Psychol. 51, 77

32 Lefebvre, L. et al. (2004) Brains, innovations and evolution in birds and primates. Brain Behav. Evol. 63, 233-246

33 Byrne, R.W. and Bates, L.A. (2007) Sociality, evolution and cognition. Curr. Biol. 17, R714-R723

34 Reader, S.M. et al. (2011) The evolution of primate general and cultural intelligence. Philos. Trans. R. Soc. B 366, 1017-1027

35 Heyes, C. (2012) What's social about social learning? J. Comp. Psychol. $126,193-202$

36 Levy, F. et al. (2003) Complete maternal deprivation affects social, but not spatial, learning in adult rats. Dev. Psychobiol. 43, 177-191

37 D'Andrea, I. et al. (2007) Communal nesting, an early social enrichment, affects social competences but not learning and memory abilities at adulthood. Behav. Brain Res. 183, 60-66

38 Branchi, I. (2009) The mouse communal nest: investigating the epigenetic influences of the early social environment on brain and behavior development. Neurosci. Biobehav. Rev. 33, 551-559
39 Moretz, J.A. et al. (2007) Behavioral syndromes and the evolution of correlated behavior in zebrafish. Behav. Ecol. 18, 556-562

40 Baron-Cohen, S. et al. (2001) The "Reading the mind in the eyes" test revised version: A study with normal adults, and adults with Asperger syndrome or high-functioning autism. J. Child Psychol. Psychiatry 42, 241-251

41 van der Maas, H.L. et al. (2006) A dynamical model of general intelligence: the positive manifold of intelligence by mutualism. Psychol. Rev. 113, 842-861

42 Arnold, S.J. (1983) Sexual selection: the interface of theory and empiricism. In Mate Choice (Bateson, P., ed.), pp. 67-107, Cambridge University Press

43 Irschick, D.J. et al. (2008) How does selection operate on wholeorganism functional performance capacities? A review and synthesis. Evol. Ecol. Res. 10, 177-196

44 McGuire, S. et al. (1999) Perceived competence and self-worth during adolescence: a longitudinal behavioral genetic study. Child Dev. 70 , 1283-1296

45 Kuo, P.H. et al. (2004) A twin study of competence and behavioral/ emotional problems among adolescents in Taiwan. Behav. Genet. 34, 63-74

46 Margulis, S.W. et al. (2005) Effects of early experience on subsequent parental behaviour and reproductive success in oldfield mice, Peromyscus polionotus. Anim. Behav. 69, 627-634

47 Auld, J.R. et al. (2010) Re-evaluating the costs and limits of adaptive phenotypic plasticity. Proc. R. Soc. Lond. B: Biol. Sci. 277, 503-511

48 Dunbar, R.I.M. and Shultz, S. (2007) Evolution in the social brain. Science 317, 1344-1347

49 Dunbar, R.I.M. (1998) The social brain hypothesis. Evol. Anthropol. 6 , 178-190

50 Chittka, L. and Niven, J. (2009) Are bigger brains better? Curr. Biol. 19, R995-R1008

51 Dukas, R. (1999) Costs of memory: ideas and predictions. J. Theor. Biol. 197, 41-50

52 Burns, J.G. et al. (2011) Costs of memory: lessons from 'mini' brains. Proc. R. Soc. Lond. B: Biol. Sci. 278, 923-929

53 Grantner, A. and Taborsky, M. (1998) The metabolic rates associated with resting, and with the performance of agonistic, submissive and digging behaviours in the cichlid fish Neolamprologus pulcher (Pisces: Cichlidae). J. Comp. Physiol. B 168, 427-433

54 Chittka, L. et al. (2009) Speed-accuracy tradeoffs in animal decision making. Trends Ecol. Evol. 24, 400-407

55 Mery, F. and Kawecki, T.J. (2003) A fitness cost of learning ability in Drosophila melanogaster.Proc. R. Soc. Lond.B:Biol.Sci. 270, 2465-2469

56 Kolss, M. and Kawecki, T.J. (2008) Reduced learning ability as a consequence of evolutionary adaptation to nutritional stress in Drosophila melanogaster. Ecol. Entomol. 33, 583-588

57 DeWitt, T.J. et al. (1998) Costs and limits of phenotypic plasticity. Trends Ecol. Evol. 13, 77-81

58 Pigliucci, M. (2005) Evolution of phenotypic plasticity: where are we going now? Trends Ecol. Evol. 20, 481-486

59 Colvis, C.M. et al. (2005) Epigenetic mechanisms and gene networks in the nervous system. J. Neurosci. 25, 10379-10389

60 Clayton, D.F. (2000) The genomic action potential. Neurobiol. Learn. Mem. 74, 185-216

61 Jarvis, E.D. et al. (1998) For whom the bird sings: context-dependent gene expression. Neuron 21, 775-788

62 Mello, C.V. et al. (1992) Song presentation induces gene expression in the songbird forebrain. Proc. Natl. Acad. Sci. U.S.A. 89, 6818

63 Dong, S. and Clayton, D.F. (2008) Partial dissociation of molecular and behavioral measures of song habituation in adult zebra finches. Genes Brain Behav. 7, 802-809

64 Mello, C.V. and Jarvis, E.D. (2008) Behavior-dependent expression of inducible genes invocal learning birds. In Neuroscience of Bird Song (Zeigler, H.P. and Marler, P., eds), pp. 381-397, Cambridge University Press

65 Burmeister, S.S. et al. (2005) Rapid behavioral and genomic responses to social opportunity. PLoS Biol. 3, e363

66 Burmeister, S.S. et al. (2008) Acoustic modulation of immediate early gene expression in the auditory midbrain of female tungara frogs. Brain Res. 1190, 105-114

67 Whitfield, C.W. et al. (2003) Gene expression profiles in the brain predict behavior in individual honey bees. Science 302, 296-299 
68 Gräff, J.et al. (2007) Differential gene expression between adult queens and workers in the ant Lasius niger. Mol. Ecol. 16, 675-683

69 Kroes, R.A. et al. (2006) Modeling depression: social dominancesubmission gene expression patterns in rat neocortex. Neuroscience 137, 37-49

70 Renn, S.C. et al. (2008) Fish and chips: functional genomics of social plasticity in an African cichlid fish. J. Exp. Biol. 211, 3041-3056

71 Machado, H.E. et al. (2009) Interspecific profiling of gene expression informed by comparative genomic hybridization: A review and a novel approach in African cichlid fishes. Integr. Comp. Biol. 49, 644-659

72 Cummings, M.E. et al. (2008) Sexual and social stimuli elicit rapid and contrasting genomic responses. Proc. R. Soc. Lond. B: Biol. Sci. 275, 393-402

73 Mukai, M. et al. (2009) Seasonal differences of gene expression profiles in song sparrow (Melospiza melodia) hypothalamus in relation to territorial aggression. PLoS ONE 4, e8182

74 Stiver, K. et al. (2004) Dispersal patterns and status change in a cooperatively breeding cichlid Neolamprologus pulcher: evidence from microsatellite analyses and behavioural observations. J. Fish Biol. 65, 91-105

75 Goodson, J.L. (2005) The vertebrate social behavior network: evolutionary themes and variations. Horm. Behav. 48, 11-22
76 Wolf, C. and Linden, D.E.J. (2012) Biological pathways to adaptability - interactions between genome, epigenome, nervous system and environment for adaptive behavior. Genes Brain Behav. 11, 3-28

77 Champagne, F.A. and Curley, J.P. (2005) How social experiences influence the brain. Curr. Opin. Neurobiol. 15, 704-709

78 Curley, J.P. et al. (2011) Social influences on neurobiology and behavior: epigenetic effects during development. Psychoneuroendocrino 36, 352371

79 Adkins-Regan, E. and Krakauer, A. (2000) Removal of adult males from the rearing environment increases preference for same-sex partners in the zebra finch. Anim. Behav. 60, 47-53

80 Branchi, I. et al. (2009) Birth spacing in the mouse communal nest shapes adult emotional and social behavior. Physiol. Behav. 96, 532539

81 Musi, B. et al. (1993) Influence of litter gender composition on subsequent maternal behaviour and maternal aggression in female house mice. Ethology 95, 43-53

82 Sharpe, R. (1975) The influence of the sex of litter-mates on subsequent maternal behaviour in Rattus norvegicus. Anim. Behav. 23, 551-559

83 Bastian, M.L. et al. (2003) Long-term effects of infant rearing condition on the acquisition of dominance rank in juvenile and adult rhesus macaques (Macaca mulatta). Dev. Psychobiol. 42, 44-51 